\title{
Characterizing and Modeling Bone Formation during Mouse Calvarial Development
}

\author{
Arsalan Marghoub, ${ }^{1}$ Joseph Libby, ${ }^{2}$ Christian Babbs, ${ }^{3}$ Yiannis Ventikos, ${ }^{1}$ Michael J. Fagan, ${ }^{2}$ and Mehran Moazen ${ }^{1, *}$ \\ ${ }^{1}$ Department of Mechanical Engineering, University College London, Torrington Place, London, WC1E 7JE, United Kingdom \\ ${ }^{2}$ Medical and Biological Engineering, School of Engineering and Computer Science, University of Hull, \\ Hull, HU6 7RX, United Kingdom \\ ${ }^{3}$ MRC Weatherall Institute of Molecular Medicine, University of Oxford, Oxford, OX3 9DS, United Kingdom
}

(Received 18 July 2018; revised manuscript received 6 November 2018; published 1 February 2019)

\begin{abstract}
The newborn mammalian cranial vault consists of five flat bones that are joined together along their edges by soft fibrous tissues called sutures. Early fusion of these sutures leads to a medical condition known as craniosynostosis. The mechanobiology of normal and craniosynostotic skull growth is not well understood. In a series of previous studies, we characterized and modeled radial expansion of normal and craniosynostotic (Crouzon) mice. Here, we describe a new modeling algorithm to simulate bone formation at the sutures in normal and craniosynostotic mice. Our results demonstrate that our modeling approach is capable of predicting the observed ex vivo pattern of bone formation at the sutures in the aforementioned mice. The same approach can be used to model different calvarial reconstruction in children with craniosynostosis to assist in the management of this complex condition.
\end{abstract}

DOI: 10.1103/PhysRevLett.122.048103

The newborn mammalian cranial vault consists of five flat bones that are joined together along their edges by soft fibrous tissues called sutures [1-4]. The sutures give flexibility for birth and allow the skull to expand and grow as the brain enlarges [3]. Sutures are composites of osteoprogenitor cells of mesenchymal origin that differentiate into osteoblasts during development. These deposit extracellular matrix consisting primarily of type I and other collagens as well as various bone-related proteins and proteoglycans [1].

During the early stages of postnatal development, hand in hand with the radial expansion of the skull, intracranial pressure (ICP) increases and calvarial bones thicken [5-8]. By the time the brain has reached its maximum size (in mice around postnatal day 20, P20 [9]) visible gaps at the sutures have reduced to micro- or nanometer gaps where the sutures have differentiated to bone $[5,10]$. The ICP has plateaued [8] while bone mineralization (i.e., both thickening of the bone and increase in its inherent mechanical properties) continues during adulthood, perhaps in response to muscle forces and mastication that started in the juvenile stages (in mouse P10-20) and continues during life [11,12].

Our understanding of the mechanobiology of the cranial sutures and the level of mechanical stimulus that sutures experience during the natural calvarial growth is still limited [13-18]. This knowledge, however, is crucial for the development of novel technologies and new approaches to the treatment of pathological conditions associated with (for example) their early fusion, i.e., craniosynostosis $[19,20]$. In this respect, laboratory mouse models are invaluable because of their genetic and morphological similarities to human calvaria [1-3]. Further, there are a number of mouse models of craniosynostosis. For example, Crouzon-type $F g f r 2^{C 342 Y}$ shows early bicoronal suture fusion causing a predictable brachycephalic head shape and bulging across the parietal region [21-24], enabling us to compare the mechanobiology of natural vs pathological bone formation at the sutures.

In a series of studies, we have previously characterized and modeled expansion of calvaria in wild-type (WT) and Crouzon-mutant-type (MT) mouse $[8,11,25]$. These have enabled us to estimate the level of mechanical strain that sutures experience during the early postnatal development. In this current work, we first characterized the changes in the calvarial sutures sizes across the skull in a series of $e x$ vivo $\mathrm{WT}$ and MT mice. Then, we developed a new modeling algorithm using the finite element method to predict bone formation at the sutures during cranial expansion. Finally, we validated our predictions through comparison with our ex vivo measurements. To the best of our knowledge, modeling the bone formation at the gross morphological level in the craniofacial system has not been carried out before. This is the novelty and main contribution of this study.

Ex vivo characterization of sutures.-We obtained microcomputed tomography (micro-CT) images of WT and MT mice at P3 ( $n=1$ for WT and MT), P7 ( $n=5$ for $\mathrm{WT}$ and MT), and P10 ( $n=5$ for WT and MT) using an X-Tek HMX 160 microCT scanner (XTek Systems Ltd, Hertfordshire, UK). Note that the mice were obtained from the same littermates. The images had a voxel size of $0.02 \mathrm{~mm}$ in all directions. We used Avizo image processing software (FEI Visualization Sciences Group, Merignac 


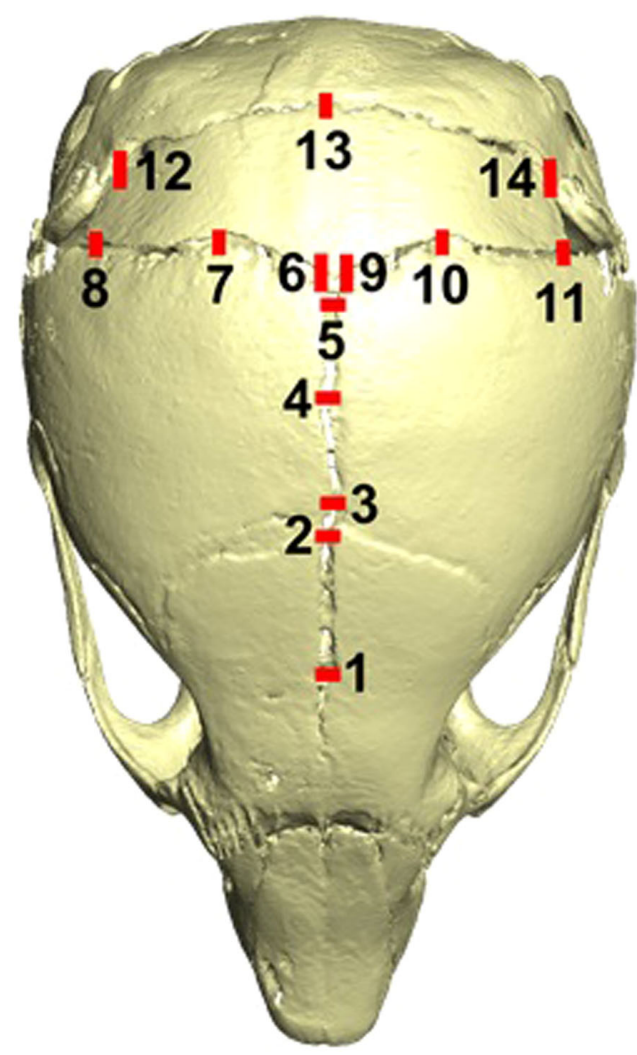

FIG. 1. Ex vivo and in silico suture sizes were measured at 14 locations: (1) frontal suture, medial point, (2) frontal suture, posterior point, (3) sagittal suture, anterior point, (4) sagittal suture, medial point, (5) sagittal suture, posterior point, (6) right interparietal suture, closest point to the midsagittal plane, (7) right interparietal suture, medial point, (8) right interparietal suture, most lateral point, (9) left interparietal suture, closest point to the midsagittal plane, (10) left interparietal suture, medial point, (11) left interparietal suture, most lateral point, (12) most lateral point of the lambdoid suture (right), (13) lambdoid suture, medial point, and (14) most lateral point of the lambdoid suture (left).

Cedex, France) to reconstruct these data into three-dimensional models. The 3D models were positioned so that in the midsagittal and transverse planes the basisphenoid and presphenoid bones were aligned with the horizontal axis. Following this alignment, we measured the suture width at 14 locations in the cranial vault (Fig. 1). At each age, we then identified the individual that was the closest average specimen for suture sizes. These specimens were then used for comparison to the computational predictions. At the same time, this characterization highlighted that the average rate of bone formation at the sutures in the WT and MT were $0.14 \mathrm{~mm}$ per day from P3 to P10.

Finite element model development.-We used micro-CT images of the WT P3 mouse to develop a three-dimensional model of the initial skull [Figs. 2(a) and 2(b)]. The geometry was first developed in Avizo and consisted of bone and sutures with an intracranial volume (ICV) that broadly represented the brain. The whole model was then transformed into a 3D solid meshed model and imported to a finite element solver, ANSYS v.18 (ANSYS Inc., Canonsburg, PA, USA). Isotropic (linear and elastic) material properties were assigned to all regions with a thermal coefficient defined only for the ICV. Bone and suture were assumed to have an elastic modulus of 3500 and $30 \mathrm{MPa}$, respectively, at age P3 [10,11]. The elastic modulus of the ICV was assumed to be $150 \mathrm{MPa}$ from a previous study [25]. The bone and suture materials were assumed to have a Poisson's ratio of 0.3 . The ICV value was 0.48 .

Boundary, interface conditions, and simulations.-The bone-suture interfaces and bone-suture-intracranial volume interfaces were assumed to be perfectly connected. Three nodes were selected on the presphenoid bone and all their degrees of freedom were constrained. The presphenoid bone was constrained because previous examination of the growth of the WT mouse skull revealed that this bone grows centrically during development and can be considered to effectively remain at the same position during the skull development (see the Supplemental Material Fig. S1 [26]). Also, we carried out several sensitivity analyses of different boundary conditions and summarized their effect on the radial expansion of the skull in a previous study [25]. Brain growth was modeled by including daily expansion of the intracranial volume, i.e., from P3 to P10, using a simple thermal expansion approach, as described previously $[25,27]$.

Modeling bone formation.-We first developed and tested our algorithm in the wild-type mouse. Here, to test for bone formation at the sutures at each step, we selected only the suture elements within a $0.1 \mathrm{~mm}$ radius of the adjacent bone [28], then if the hydrostatic strain within any element was smaller than 5\% [29,30], its modulus of elasticity was increased by $250 \mathrm{MPa}$ (from $30 \mathrm{MPa}$ ). The choice of $250 \mathrm{MPa}$ was based on our previous study of quantifying bone properties on same mouse model [11]; briefly, this was estimated based on a linear extrapolation between the calvarial properties at P10 and P20. Then, the skull shape, i.e., the geometry, was updated and the elastic modulus of the bone was increased by $250 \mathrm{MPa}$ in preparation for the next step (or age or day of development). No adaptive remeshing algorithm was used here, as the geometry was updated day by day to the new deformed shape. This approach avoided element distortions that would have otherwise occurred due to the large deformation.

The same remodeling process was then repeated; i.e., new suture elements (with $E=30 \mathrm{MPa}$ ) were selected at the edges of the remodeled tissue at the end of the previous step. The model was loaded, and pending the level of hydrostatic strain within the selected suture elements, their elastic modulus was altered (i.e., increased from $E=30 \mathrm{MPa}$ to $E=280 \mathrm{MPa}$ ). Here, before increasing the modulus of elasticity of the sutures, the modulus of the 


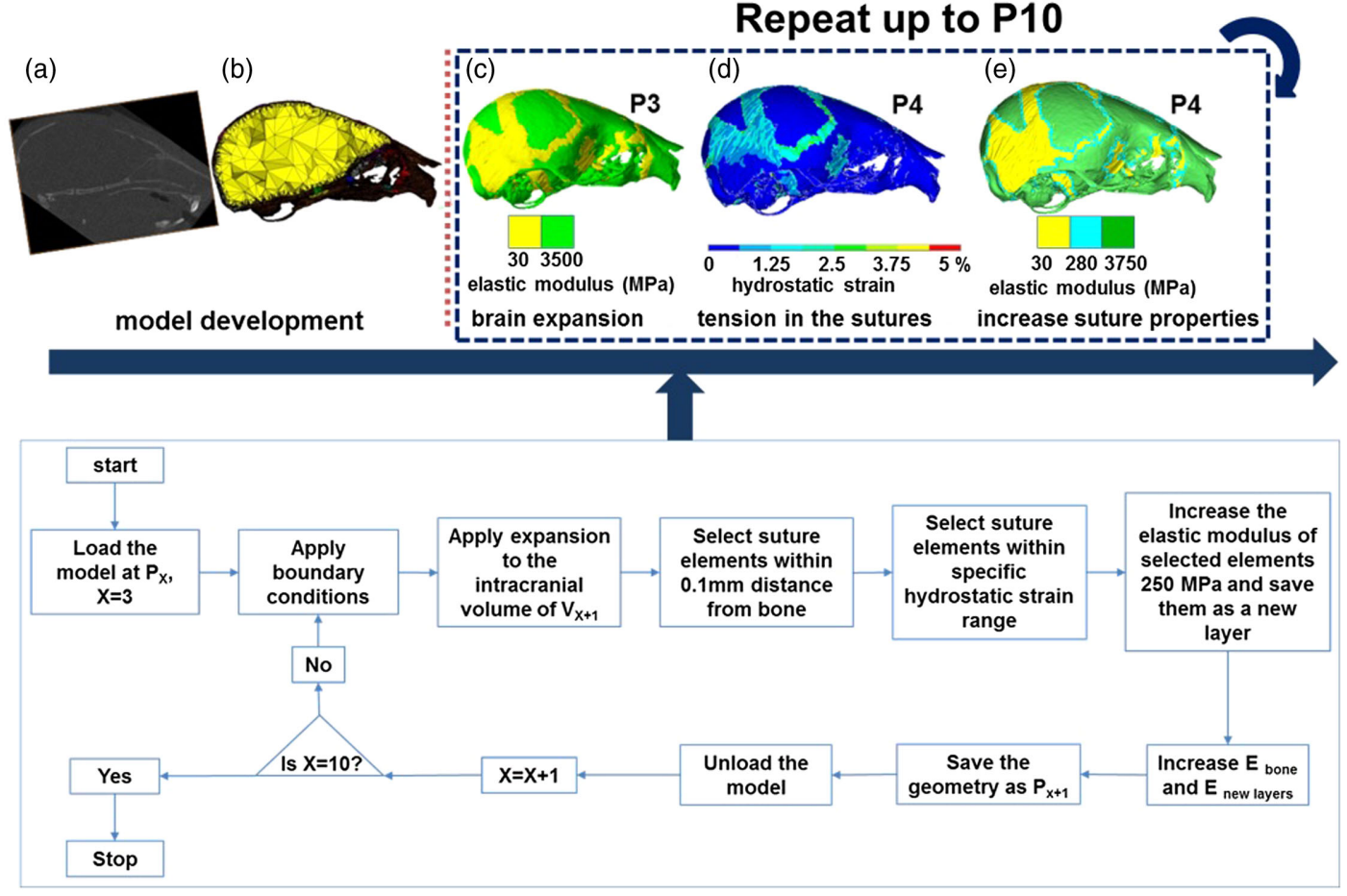

FIG. 2. Using microCT images of a WT mouse skull at P3 (a), a 3D finite element model was developed (b). After assigning the material properties and applying boundary conditions (c), the intracranial volume was expanded to the volume of the next age, i.e., at P4 (d). Material properties of the bone and the suture elements within the specified hydrostatic strain range and distance from the bone were updated (e). This process was repeated until P10. The flow diagram shows the overall process.

previously remodeled layer (with $E=280 \mathrm{MPa}$ ), was increased by a further 250 to $530 \mathrm{MPa}$. Then, similar to the previous step, the skull shape was updated and the same process was repeated up to P10 (see Fig. 2). Note that the interfaces between the different layers of materials that formed throughout the tissue differentiation process (i.e., as age increased day by day) were fixed. In other words, these elements shared the same nodes and only their modulus of elasticity was altered.
We carried out a series of detailed sensitivity analyses to test the choice of strain, i.e., comparing hydrostatic strain, von Mises strain, first principal strain, the strain range, and selection radius. The results of these sensitivity analyses are included in the Supplemental Material Fig. S2 [26].

Predicting bone formation in the mutant F $\mathrm{Ff} 2^{\mathrm{C} 342 \mathrm{Y} /+}$ mouse at P10.-Using the baseline WT model and initially fusing some of the sutures based on the study of Liu et al. [23] and our own observation, the bone formation in the

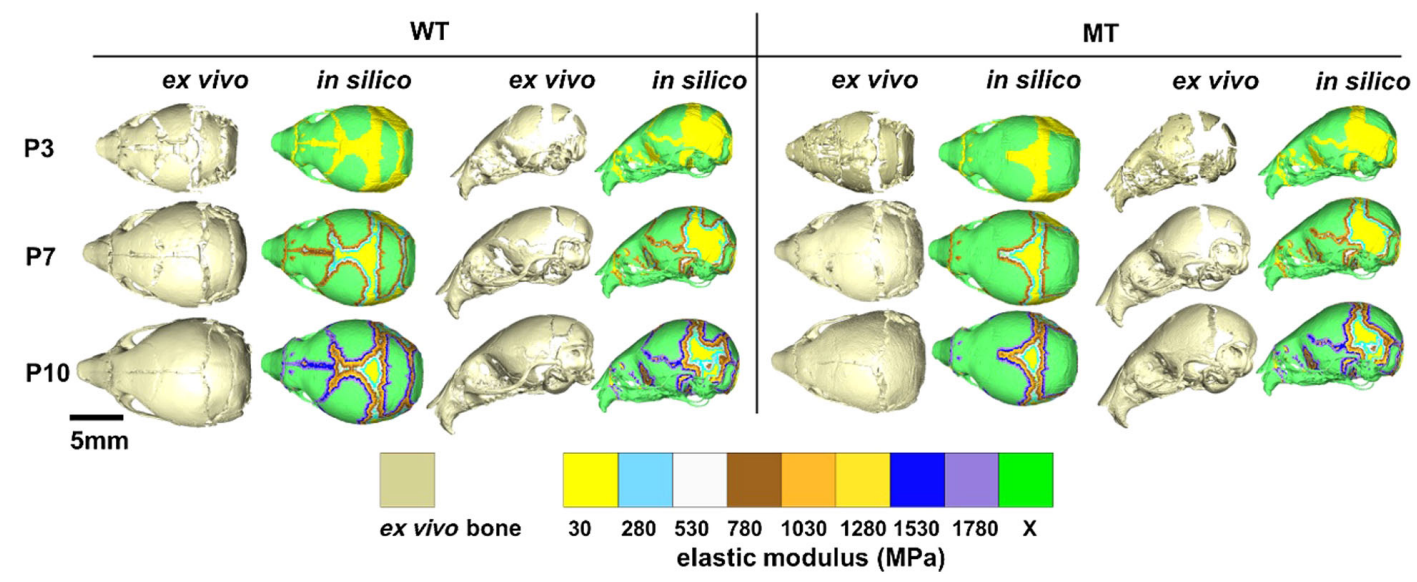

FIG. 3. WT and MT ex vivo and in silico skull and suture size changes from P3 to P10. In silico images show the tissue differentiation as skull grows. Note that the elastic modulus of the bone tissue, i.e., green areas $(X)$ is increased by $250 \mathrm{MPa}$ at the end of each age, from $3500 \mathrm{MPa}$ at P3 to $5250 \mathrm{MPa}$ at P10. 


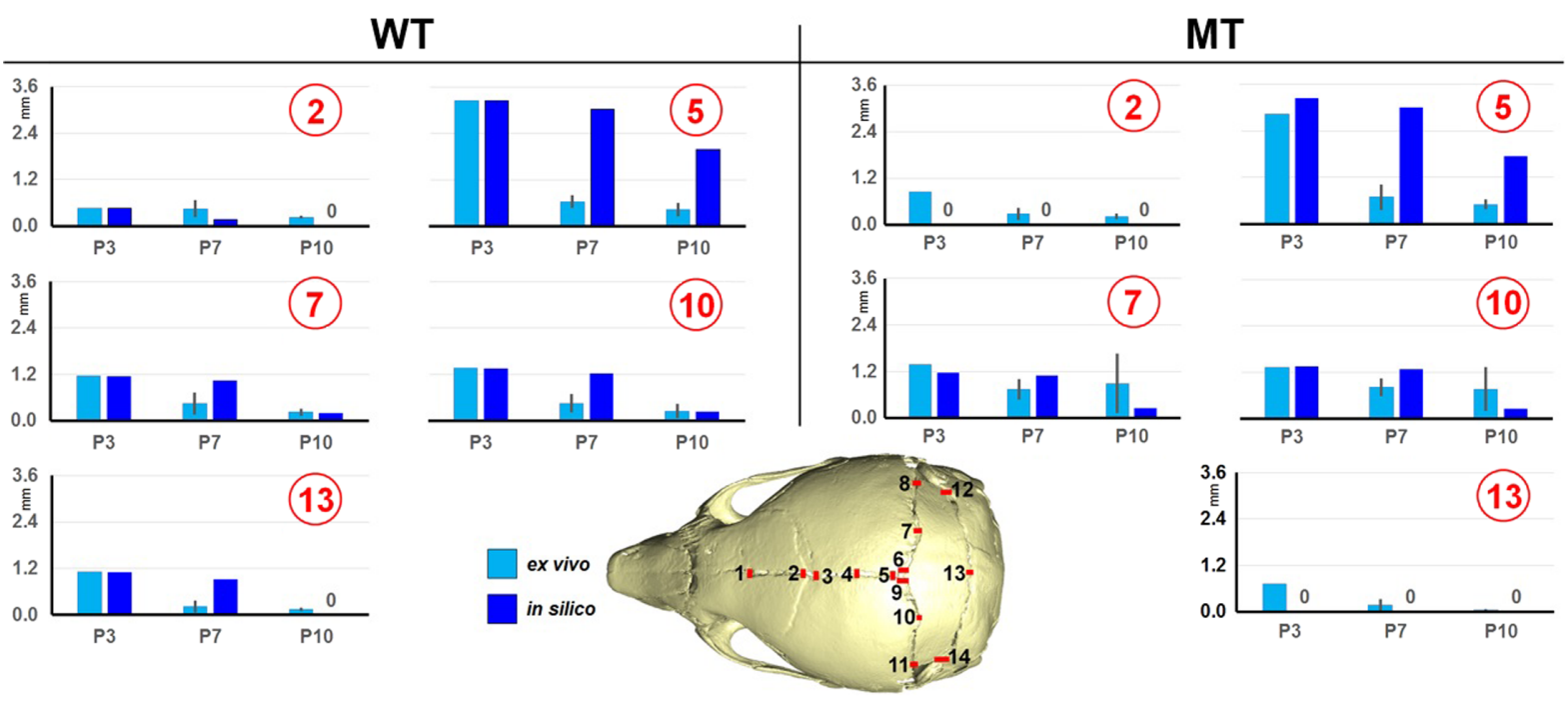

FIG. 4. Bone formation comparison between ex vivo and in silico models at five measuring points. Note that in MT, in silico model points 2 and 13 suture sizes are 0 at all ages because they have been considered to be fused prematurely.

mutant skull at P10 was predicted. During the analysis, the elastic modulus of frontal, coronal, lambdoid, and presphenoid-basisphenoid synchondrosis (PBS) sutures were changed from 30 to $3500 \mathrm{MPa}$ (i.e., the same as bone) to model the premature fusion of these sutures in the mutant model. The expansion rate was kept similar to the WT model, and the results were compared to the values from the microCT data of the MT mice at P10.

Measurements and comparison.-Figure 3 shows a comparison of our predictions of the suture closure at $\mathrm{P} 7$ and P10 with the ex vivo suture measurements for both WT and MT mice. Our simulation captures the overall pattern of bone formation across the skull. For example, it predicts the fusion of the posterior frontal suture by day ten, while the sagittal suture and posterior fontanel remain open. Similarly, in the mutant mice, anterior-posterior closure of the sagittal suture was comparable to our $e x$ vivo findings. See also the Supplemental Material Fig S3 [26] for the WT results at P7 and P10 with different scale bar, i.e., highlighting predicted mineralized tissue with the elastic modulus above $1000 \mathrm{MPa}$.

In Fig. 4, we compare sample suture sizes from our simulations at five regions out of the 14 regions identified in Fig. 1. A full comparison between the ex vivo results and our prediction is provided in Table I. In this analysis, it is assumed that tissues with an elastic modulus equal to or less than $780 \mathrm{MPa}$ (based on our predictions) are still suture, while tissues with elastic modulus greater than $780 \mathrm{MPa}$ are bone or hard tissue (see the Supplemental Material Fig. S3 [26]). Our model predicts a gradual reduction in suture sizes across all the sutures from P3 to P10. It should be noted that, in the mutant model at regions 2 and 13, the sutures were assumed to be fused at P3 to mimic the early fusion of these sutures in the Crouzon mouse. Hence, our predictions at P7 and P10 were also a fused suture, whereas our ex vivo measurements show that these regions are not fully fused at the aforementioned ages.

Discussion.-There is no doubt that there is a complex mix of chemical and biological signaling that regulates bone formation at the sutures. Several previous studies have

TABLE I. Comparison between the ex vivo and in silico (FE) suture size changes $(\mathrm{mm})$ in WT and mutant-type mouse during the development (i.e., at postnatal [P] day three, seven, and ten) at 14 locations. The frontal, coronal, lambdoid, and PBS sutures were fused prematurely at P3 for the MT FE model; hence the suture sizes at points $1,2,12,13$, and 14 are $0 \mathrm{~mm}$ from $\mathrm{P} 3$ to $\mathrm{P} 10$. *SD at P3 for both WT and MT is zero due to having only one sample of each. Note that "Av" is "average"; "SD" is "standard deviation."

\begin{tabular}{|c|c|c|c|c|c|c|}
\hline & \multicolumn{6}{|c|}{ P3 } \\
\hline & \multicolumn{3}{|c|}{ WT } & \multicolumn{3}{|c|}{ MT } \\
\hline & Av ex vivo & $\mathrm{SD}^{*}$ & $\mathrm{FE}$ & Av ex vivo & $\mathrm{SD}^{*}$ & $\mathrm{FE}$ \\
\hline 1 & 0.63 & 0.00 & 0.63 & 1.03 & 0.00 & 0.00 \\
\hline 2 & 0.46 & 0.00 & 0.46 & 0.84 & 0.00 & 0.00 \\
\hline 3 & 1.20 & 0.00 & 1.20 & 1.88 & 0.00 & 1.20 \\
\hline 4 & 1.46 & 0.00 & 1.46 & 1.41 & 0.00 & 1.46 \\
\hline 5 & 3.24 & 0.00 & 3.24 & 2.84 & 0.00 & 3.24 \\
\hline 6 & 1.11 & 0.00 & 1.11 & 1.86 & 0.00 & 1.11 \\
\hline 7 & 1.16 & 0.00 & 1.16 & 1.37 & 0.00 & 1.16 \\
\hline 8 & 1.24 & 0.00 & 1.24 & 1.40 & 0.00 & 1.24 \\
\hline 9 & 1.10 & 0.00 & 1.10 & 1.58 & 0.00 & 1.10 \\
\hline 10 & 1.36 & 0.00 & 1.36 & 1.33 & 0.00 & 1.36 \\
\hline 11 & 1.41 & 0.00 & 1.41 & 1.24 & 0.00 & 1.41 \\
\hline 12 & 1.53 & 0.00 & 1.53 & 1.16 & 0.00 & 0.00 \\
\hline 13 & 1.11 & 0.00 & 1.11 & 0.73 & 0.00 & 0.00 \\
\hline 14 & 1.30 & 0.00 & 1.30 & 0.91 & 0.00 & 0.00 \\
\hline
\end{tabular}

(Table continued) 
TABLE I. (Continued)

\begin{tabular}{|c|c|c|c|c|c|c|}
\hline & \multicolumn{6}{|c|}{ P7 } \\
\hline & \multicolumn{3}{|c|}{ WT } & \multicolumn{3}{|c|}{ MT } \\
\hline & Av ex vivo & SD & $\mathrm{FE}$ & Av ex vivo & SD & $\mathrm{FE}$ \\
\hline 1 & 0.53 & 0.17 & 0.56 & 0.38 & 0.18 & 0.00 \\
\hline 2 & 0.45 & 0.21 & 0.17 & 0.28 & 0.16 & 0.00 \\
\hline 3 & 0.61 & 0.07 & 1.03 & 0.36 & 0.17 & 0.57 \\
\hline 4 & 0.42 & 0.11 & 1.22 & 0.40 & 0.29 & 1.12 \\
\hline 5 & 0.64 & 0.17 & 3.03 & 0.69 & 0.33 & 3.00 \\
\hline 6 & 0.73 & 0.30 & 1.07 & 1.13 & 0.24 & 1.16 \\
\hline 7 & 0.44 & 0.28 & 1.05 & 0.74 & 0.27 & 1.10 \\
\hline 8 & 0.69 & 0.21 & 1.10 & 0.67 & 0.16 & 1.15 \\
\hline 9 & 0.80 & 0.24 & 1.06 & 0.89 & 0.17 & 1.11 \\
\hline 10 & 0.46 & 0.24 & 1.23 & 0.83 & 0.23 & 1.28 \\
\hline 11 & 0.71 & 0.30 & 1.34 & 0.56 & 0.32 & 1.45 \\
\hline 12 & 0.51 & 0.24 & 1.36 & 0.36 & 0.13 & 0.00 \\
\hline 13 & 0.22 & 0.15 & 0.93 & 0.17 & 0.13 & 0.00 \\
\hline \multirow[t]{4}{*}{14} & 0.55 & 0.16 & 0.95 & 0.46 & 0.17 & 0.00 \\
\hline & \multicolumn{6}{|c|}{ P10 } \\
\hline & \multicolumn{3}{|c|}{ WT } & \multicolumn{3}{|c|}{ MT } \\
\hline & Av ex vivo & SD & $\mathrm{FE}$ & $\mathrm{Av}$ ex vivo & SD & $\mathrm{FE}$ \\
\hline 1 & 0.39 & 0.13 & 0.00 & 0.36 & 0.22 & 0.00 \\
\hline 2 & 0.23 & 0.03 & 0.00 & 0.21 & 0.07 & 0.00 \\
\hline 3 & 0.31 & 0.09 & 0.66 & 0.27 & 0.05 & 0.00 \\
\hline 4 & 0.32 & 0.15 & 0.25 & 0.32 & 0.13 & 0.14 \\
\hline 5 & 0.43 & 0.18 & 1.99 & 0.50 & 0.13 & 1.74 \\
\hline 6 & 0.33 & 0.13 & 0.66 & 0.64 & 0.48 & 0.69 \\
\hline 7 & 0.22 & 0.10 & 0.21 & 0.88 & 0.77 & 0.24 \\
\hline 8 & 0.31 & 0.14 & 0.25 & 0.49 & 0.30 & 0.28 \\
\hline 9 & 0.45 & 0.25 & 0.71 & 0.60 & 0.27 & 0.77 \\
\hline 10 & 0.26 & 0.19 & 0.24 & 0.77 & 0.56 & 0.26 \\
\hline 11 & 0.49 & 0.27 & 1.04 & 0.52 & 0.26 & 1.09 \\
\hline 12 & 0.37 & 0.22 & 1.03 & 0.33 & 0.13 & 0.00 \\
\hline 13 & 0.14 & 0.04 & 0.00 & 0.04 & 0.06 & 0.00 \\
\hline 14 & 0.48 & 0.24 & 0.58 & 0.19 & 0.07 & 0.00 \\
\hline
\end{tabular}

suggested that mechanical strain must also be a key factor (e.g., Refs. $[14,16])$. Here, we developed a new algorithm to model this phenomenon based on mechanical strain experienced by the sutures during the skull growth.

A number of assumptions and approximations had to be made in the simulations, but still there was good agreement in the pattern of bone formation across the sutures and with the ex vivo results. Perhaps the most significant were the following: (1) The sutures were modeled as a linear elastic material while they are known to be nonlinear and viscoelastic. Given the timescale on which our simulations were performed here, i.e., growth over ten days, we think that perhaps a linear model could be acceptable. (2) Uniform bone deposition was assumed at all sutures. It is possible that different sutures may have different bone deposition rate. Including such a rate-dependent bone deposition might indeed address some of the discrepancies that we observed (Fig. 3). (3) Bone formation is a complex mix of various biological and nonbiological factors; however, our approach is to model the bone formation purely based on the level of hydrostatic strain. While this is indeed a huge simplification, the radius of bone formation that was specified in our approach (i.e., $0.1 \mathrm{~mm}$ bone formation rate) implicitly takes into account these complex factors through a combined macroscopic effect. Nonetheless, further work possibly should be undertaken to explicitly incorporate the various signaling events into the approach developed here and to address the other limitations of this work.

We were not able to validate the tissue differentiation that our model predicted at the sutures, which will require further experimental measurements (see, e.g., Ref. [31]). Nonetheless, our previous nanoindentation of bone in mouse models with the same genetic background showed a lower elastic modulus in bone adjacent to the sutures [11]. This is similar to our current predictions but in a qualitative fashion rather than a more quantitative analysis. See also our previous study for a detailed quantitative morphological comparison between the FE prediction of skull shape at P10 with an "average" ex vivo wild-type and Crouzon mouse at P10 [25].

In summary, we think the modeling approach presented here has potential in the modeling of calvarial growth. This could provide significant advancement in terms of comparing different reconstruction methods for the treatment of craniosynostosis and understanding the optimum management of various forms of this condition [32], which in the long term could reduce the complications currently associated with the treatment of craniosynostosis.

This work was supported by the Royal Academy of Engineering (Grant No. 10216/119 to M. M.). The authors thank Andrew Wilkie, Erwin Pauws, and David Johnson for their advice and support throughout this study.

*Corresponding author.

M.Moazen@ucl.ac.uk

[1] L. A. Opperman, Dev. Dyn. 219, 472 (2000).

[2] G. Morriss-Kay and A. O. M. Wilkie, J. Anat. 207, 637 (2005).

[3] J. T. Richtsmeier and K. Flaherty, Acta Neuropathol. 125, 469 (2013).

[4] S. W. Jin, K. B. Sim, and S. D. Kim, J. Korean Neurol. Soc. 59, 192 (2016).

[5] M. L. Moss, Am. J. Anat. 94, 333 (1954).

[6] A. S. Dekaban, Annals of Neurology 2, 485 (1977).

[7] S. S. Margulies and K. L. Thibault, J. Biomech. Eng. 122, 364 (2000).

[8] M. Moazen, A. Alazmani, K. Rafferty, Z. J. Liu, J. Gustafson, M. L. Cunningham, M. J. Fagan, and S. W. Herring, J. Biomech. 49, 123 (2016).

[9] M. Aggarwal, J. Zhang, M. I. Miller, R. L. Sidman, and S. Mori, Neuroscience (N.Y.) 162, 1339 (2009). 
[10] J. H. Henderson, L. Y. Chang, H. M. Song, M. T. Longaker, and D. R. Carter, J. Biomech. 38, 2294 (2005).

[11] M. Moazen, E. Peskett, C. Babbs, E. Pauws, and M. J. Fagan, PLoS One 10, e0125757 (2015).

[12] K. L. Rafferty and S. W. Herring, J. Morphol. 242, 167 (1999).

[13] J. J. Mao, J. Dent. Res. 81, 810 (2002).

[14] D. R. Carter and G. S. Beaupre, Skeletal Function and Form: Mechanobiology of Skeletal Development, Aging, and Regeneration (Cambridge University Press, Cambridge, England, 2007).

[15] S. W. Herring, Front. Oral Biol. 12, 41 (2008).

[16] J. Weickenmeier, C. Fischer, D. Carter, E. Kuhl, and A. Goriety, Phys. Rev. Lett. 118, 248101 (2017).

[17] C. Lee, J. T. Richtsmeier, and R. H. Kraft, J. Mech. Behav. Biomed. Mater. 17, 1750073 (2017).

[18] R. H. Khonsari, J. Olivier, P. Vigneaux, S. Sanchez, P. Tafforeau, P. E. Ahlberg, F. Di Rocco, D. Bresch, P. Corre, A. Ohazama, P. T. Sharpe, and V. Calvez, Proc. R. Soc. B 280, 20122670 (2013).

[19] K. Flaherty, N. Singh, and J. T. Richtsmeier, Wiley Interdiscip. Rev. Dev. Biol. 5, 429 (2016).

[20] Z. Al-Rekabi, M. L. Cunningham, and N. J. Sniadecki, ACS Biomater. Sci. Eng. 3, 2733 (2017).

[21] M. Grova, D. D. Lo, D. Montoro, J. S. Hyun, M. T. Chung, D. C. Wan, and M. T. Longaker, J. Craniofac. Surg. 23, S12 (2012).
[22] V. P. Eswarakumar, M. C. Horowitz, R. Locklin, G. M. Morriss-Kay, and P. Lonai, Proc. Natl. Acad. Sci. U.S.A. 101, 12555 (2004).

[23] J. Liu, H. K. Nam, E. Wang, and N. E. Hatch, Calcif. Tissue Int. 92, 451 (2013).

[24] E. Peskett, S. Kumar, W. Baird, J. Jaiswal, M. Li, P. Patel, J. A. Britto, and E. Pauws, Biol. Open 6, 223 (2017).

[25] A. Marghoub, J. Libby, C. Babbs, E. Pauws, M. J. Fagan, and M. Moazen, J. Anat. 232, 440 (2018).

[26] See Supplemental Material at http://link.aps.org/ supplemental/10.1103/PhysRevLett.122.048103 for further details and several sensitivity analyses.

[27] J. Libby, A. Marghoub, D. Johnson, R. Khonsari, M. J. Fagan, and M. Moazen, J. R. Soc. Interface 14, 20170202 (2017).

[28] J. H. Henderson, M. T. Longaker, and D. R. Carter, Bone (N.Y.) 34, 271 (2004).

[29] L. E. Claes and C. A. Heigele, J. Biomech. 32, 255 (1999).

[30] M. Doblaré and J. M. García-Aznar, Arch. Comput. Methods Eng. 13, 471 (2006).

[31] P. L. Leong and E. F. Morgan, Acta Biomater. 4, 1569 (2008).

[32] O. Malde, J. Libby, and M. Moazen, Mol. Syndromol. 10, 74 (2019). 